Glosse

\title{
Für Ärzte wird alles besser
}

Endlich einmal frohe Botschaften aus der Medizin. Eigentlich ist die offiziell verordnete Zwangsgesinnung in diesem System ja von Pessimismus geprägt. Es fehlt praktisch an allem. Da überrascht es schon sehr, wenn, wie beim diesjährigen Internistenkongress, die Zukunft der Medizin durchaus optimistisch gesehen wird. Aus Sicht der Ärzte wird alles besser, ja sogar wunderbar. Während im Rentensystem schon länger die Alarmglocken läuten, da immer weniger Arbeitswillige immer mehr Rentner ernähren müssen, ist es in der Medizin genau umgekehrt. Während heute nur 500 Patienten einen Arzt ernähren müssen, werden es in 5 Jahren bereits 1.000 Patienten sein und, wenn diese erfreuliche Entwicklung anhält, bald sogar 2.000. Wo letztlich die Grenze liegen wird, bei der man die Zahl der Patienten begrenzen muss, damit Ärzte nicht überversorgt sind, weiß im Moment niemand genau. Damit sind die Existenz der Ärz-

te und hoffentlich auch stolze jährliche Honorarzuwächse gesichert ...

Dazu kommt noch etwas Erfreuliches. Die moderne Informationstechnologie wird den Arzt in Zukunft vor allerlei unliebsamen Routinetätigkeiten befreien. Er muss sich nicht mehr mit Banalitäten herumärgern. Apps können nämlich Blutdruck, Puls, Köpertemperatur und vieles andere messen und, wenn man die Symptome eingibt, wird sogar eine zuverlässige Diagnose gestellt und eine Therapiempfehlung gegeben. Dazu kommen Anleitungen übers Internet, wie man kleinere operative Eingriffe an sich selbst ohne Risiken und Komplikationen durchführen kann. Bei größeren Operationen müssen Familienangehörige entsprechend geschult werden, heute alles kein Problem mehr. So wird jeder zum Arzt in eigener Sache. Wenn das keine individualisierte Medizin ist!

\section{Dr. Peter Stiefelhagen}

\section{Walter-Trummert-Preis an Friederike Klein}

\section{Autorin der MMW ausgezeichnet}

Der Verband der Medizin- und Wissenschaftsjournalisten e.V. (VMWJ) verleiht seit 1974 den Walter-Trummert-

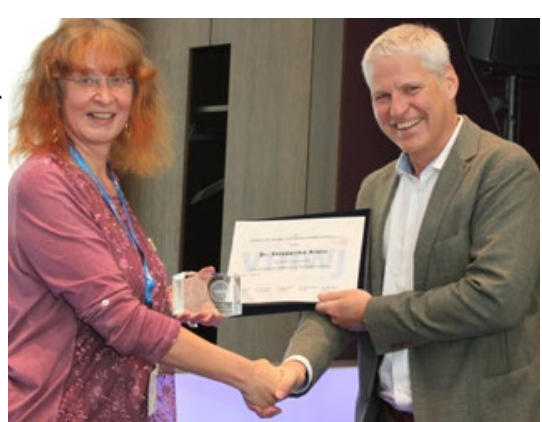

Laudator Dr. Dirk Einecke vom VMWJVorstand gratuliert der Preisträgerin.
Preis an verdiente Medizinjournalisten. In diesem Jahr ging der Preis an Friederike Klein, Journalistin aus München.

Nach dem Studium der Biologie begann Friederike Klein ihre berufliche Karriere 1990 als Lektorin. Seit 1999 verantwortete sie im Verlag Urban \& Vogel den Bereich Facharzt- und Pflege-Zeitschriften sowie Kongresse. Seit 2005 arbeitet sie als freie Medizinjournalistin, wo sie ein breites Themenspektrum abdeckt und auch wiederholt für die MMW tätig ist. $\mathrm{Zu}$ ihren besonderen Kompetenzen gehört es, Veranstaltungen zu moderieren und den journalistischen Nachwuchs auszubilden.
Post-Lyme-Syndrom

\section{Langzeit-Antibiose sinnlos}

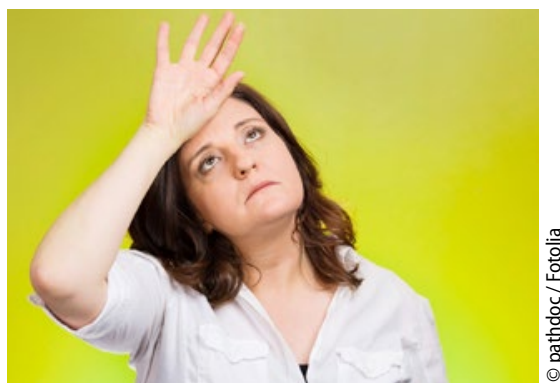

V.a. Post-Lyme-Syndrom: Sind wirklich Borrelien Schuld an ihren Beschwerden?

Die wochen- oder gar monatelange Behandlung mit Antibiotika nützt nichts beim Post-Lyme-Syndrom. Das hat eine randomisiert-kontrollierte Studie aus den Niederlanden bestätigt. Behandelt wurden 280 Patienten, bei denen entweder in der Vergangenheit eine Borreliose diagnostiziert worden war oder bei denen sich zeitgleich mit den Beschwerden Immunoblot-bestätigt IgG- und IgM-Antikörper auf Borrelien nachweisen ließen. Fast alle Patienten klagten über Arthralgien, Fatigue, muskuloskelettale Schmerzen sowie neurokognitive und sensorische Störungen. Alle bekamen zunächst für zwei Wochen 2 g/d Ceftriaxon i.v., ein Drittel erhielt anschließend zwölf Wochen lang Doxycyclin (100 mg 2x täglich), ein weiteres Drittel Clarithromycin/Hydroxychloroquin (CHC, $500 \mathrm{mg}+200 \mathrm{mg}$ $2 \mathrm{x}$ täglich), die übrigen Placebo.

Nach 14 Wochen gab es zwischen den Gruppen keinerlei signifikante Unterschiede bezüglich der verschiedenen Beschwerden. In der Doxycyclin-Gruppe klagte jedoch jeder Fünfte über Photosensitivität und jeder Zehnte über Übelkeit. Unter CHC traten Übelkeit und Rush häufiger auf als mit Placebo.

$$
\text { mut }
$$

- Berende A et al. NEngl J Med 2016;374:1209-20

$\rightarrow$ http://www.awmf.org/uploads/tx_szleitlinien/030-0711 S1_Neuroborreliose_2012_verlaengert.pdf 